\title{
MammoSys: A content-based image retrieval system using breast density patterns
}

\author{
Júlia E.E. de Oliveira ${ }^{a, *}$, Alexei M.C. Machado ${ }^{b}$, Guillermo C. Chavez ${ }^{a}$, \\ Ana Paula B. Lopes ${ }^{a}$, Thomas M. Deserno ${ }^{c}$, Arnaldo de A. Araújo ${ }^{a}$ \\ a Universidade Federal de Minas Gerais, Departamento de Ciência da Computação, Av. Antônio Carlos, 6627, \\ 31270-901, Belo Horizonte, MG, Brazil \\ b Pontifícia Universidade Católica de Minas Gerais, Departamento de Ciência da Computaçãão, Instituto de Informática, R. Dom Jose \\ Gaspar, 500 - Prédio 34, sala 215, 30535-610, Belo Horizonte, MG, Brazil \\ c Aachen University of Technology (RWTH), Department of Medical Informatics, Pauwelsstr. 30, D-52057 Aachen, Germany
}

\section{A R T I C L E I N F O}

Article history:

Received 14 August 2009

Received in revised form

19 January 2010

Accepted 21 January 2010

\section{Keywords:}

Medical images

Breast density

Content-based image retrieval

Two-dimensional principal

component analysis

Support vector machine

\begin{abstract}
A B S T R A C T
In this paper, we present a content-based image retrieval system designed to retrieve mammographies from large medical image database. The system is developed based on breast density, according to the four categories defined by the American College of Radiology, and is integrated to the database of the Image Retrieval in Medical Applications (IRMA) project, that provides images with classification ground truth. Two-dimensional principal component analysis is used in breast density texture characterization, in order to effectively represent texture and allow for dimensionality reduction. A support vector machine is used to perform the retrieval process. Average precision rates are in the range from $83 \%$ to $97 \%$ considering a data set of 5024 images. The results indicate the potential of the system as the first stage of a computer-aided diagnosis framework.
\end{abstract}

@ 2010 Elsevier Ireland Ltd. All rights reserved.

\section{Introduction}

Breast density has been shown to be related to the risk of developing breast cancer [1] since women with dense breast tissue can hide lesions, causing cancer to be detected at later stages. The BI-RADS (Breast Imaging Reporting Data System) density scale, developed by the American College of Radiology, ${ }^{1}$ informs radiologists about the decline in sensitivity of mammography with increasing breast density. BI-RADS defines density I as almost entirely fatty, density II as scattered fibroglandular tissue, density III as heterogeneously dense tissue, and IV as extremely dense tissue.

Mammography is the main screening tool for cancer and radiologists evaluate and report breast density on the basis of visual analysis of mammographies. The various distribution of the parenchyma tissue makes automatic classification a difficult task, so in this scenario, as auxiliary tools, computer-aided diagnosis (CAD) and content-based image retrieval (CBIR) systems appear as real possibilities to help radiologists reduce the variability of their analysis and also improve the accuracy of

\footnotetext{
* Corresponding author. Tel.: +55 3125358248.

E-mail addresses: julia@dcc.ufmg.br (J.E.E. de Oliveira), alexei@pq.cnpq.br (A.M.C. Machado), gcamarac@gmail.com (G.C. Chavez), paula@dcc.ufmg.br (A.P.B. Lopes), tdeserno@mi.rwth-aachen.de (T.M. Deserno), arnaldo@dcc.ufmg.br (A.d.A. Araújo).

1 http://www.acr.org. 0169-2607/\$ - see front matter @ 2010 Elsevier Ireland Ltd. All rights reserved. doi:10.1016/j.cmpb.2010.01.005
} 
Table 1 - Summary of published works based on the analysis of breast density, with the developed system, number of mammographies, features, and classifiers used in the studies.

$\begin{array}{lllll}\text { Author } & \text { Kinoshita [8] } & \text { Oliver [9] } & \text { Castella [12] } & \text { Wang [13] } \\ \text { Year } & 2007 & 2008 & 2007 & \text { C003 } \\ \text { System } & \text { CBIR } & \text { CAD } & \text { CAD } & \text { CAD } \\ \text { No. of images } & 1080 & 1155 & 352 & \text { Fractal, } \\ \text { Features } & \text { Shape, } & & \text { Primitive, } & \text { Histogram } \\ & \text { Moments, } & \text { Morphology, } & \text { Histogram, } \\ & \text { Granulometry, } & \text { Texture } & \text { Texture, } \\ & \text { Histogram, } & & \text { NGTDM } \\ & \text { Texture, } & & & \text { Bayes, } \\ \text { Radon } & & \text { LDA, } & \text { Neural network } \\ \text { Classifier } & \text { SOM } & \text { k-NN, } & \text { Necision tree, } & \\ & & \text { Combined Bayesian } & \end{array}$

mammography interpretation. CBIR systems [2,3], which are a component of CAD systems, use visual information extracted from images to retrieve similar images to a specific query. A CBIR system does not need to provide diagnostic information related to the retrieved images but just present similar images according to a certain pattern. Therefore, a CBIR system based on breast density, from a clinical point of view, may guide the radiologist on the detection of a lesion and its classification. Moreover, this system is the first step, and a very important one, for the development of a CAD system.

In this paper, we propose, implement, and evaluate a CBIR system called MammoSys. A contribution of this work is to introduce the two-dimensional principal component analysis (2DPCA) method [4] for the characterization of breast density texture, that allows for feature extraction at the same time that dimensionality reduction is performed. Two-dimensional principal component analysis overcomes principal component analysis (PCA) as it is simpler and more straightforward to use for image feature extraction since 2DPCA is directly applied to the image matrix. Retrieval is performed with the aid of a support vector machine (SVM) [5], that is able to solve a variety of learning, classification, and prediction problems.

The appropriate characterization of images together with the storage and management of the large amount of images produced by hospitals and medical centers are a main challenge in the development of CBIR systems. The IRMA (Image Retrieval in Medical Applications) project ${ }^{2}[6]$ deals with this kind of problems, as it aims at developing and implementing high-level methods for CBIR systems with prototypal application to medico-diagnostic tasks on radiological image archiving. The database has more than 10,000 mammographies [7], all of them with available ground truth information that offers invaluable support to the validation of the method proposed in this work.

The remainder of this paper is divided into seven sections. Section 2 provides a brief summary of related works. Section 3 introduces the texture characterization of breast tissue through 2DPCA. In Section 4, we explain the principles of the SVM classifier used for the retrieval task. Section 5 describes the experiments designed to evaluate the proposed model. In Section 6, we present and discuss results, and in Section 7, we state the conclusion of this work.

\footnotetext{
${ }^{2}$ http://www.irma-project.org.
}

\section{Background}

In the context of mammography and breast density, some works have explored the use of CBIR and CAD systems to improve knowledge and provide facilities on these modalities. Table 1 summarizes some of the published works based on breast density for classification and retrieval.

In the work of Kinoshita et al. [8], breast density is used as a pattern to retrieve images from a 1080 mammography database, available at the Clinical Hospital of the University of São Paulo at Ribeirão Preto, Brazil. Shape descriptors, texture features, moments, Radon transform, granulometry, and histograms were used to characterize breast density, and the Kohonen self-organizing map (SOM) neural network was used for the retrieval task. Precision rates between 79\% and $83 \%$ were obtained for $50 \%$ of recall and precision rates between $79 \%$ and $86 \%$ were obtained considering the first $25 \%$ of the retrieved images. Despite the fact that these results indicated effective retrieval, based on features such as histograms and shape, the authors concludes that additional studies would be required in order to improve all the process.

In the field of CAD systems, Oliver et al. [9] characterized breast densities using morphological and texture features in order to propose a CAD system for breast density classification. A set of 322 mammographies was obtained from MIAS (The Mammographic Image Analysis Society Digital Mammogram Database $)^{3}[10]$ database and 833 mammographies were obtained from DDSM (Digital Database for Screening Mammography) ${ }^{4}[11]$ database. A decision tree classifier, $k$ nearest neighbor, and a combined Bayesian classification were used for classification and the best results were around $82 \%$ of correct classification for the MIAS database, and 77\% for DDSM database.

Also for breast density classification, Castella et al. [12] developed a semi-automatic method using 352 mammographies from Clinique des Grangette at Geneva, Switzerland. Breast density was characterized through texture features, gray level histograms, primitives, neighborhood gray tone difference matrix (NGTDM), and fractal analysis. The images were classified using Bayes classifier, Naïve Bayes classifier,

\footnotetext{
${ }^{3}$ http://peipa.essex.ac.uk/ipa/pix/mias.

${ }^{4}$ http://marathon.csee.usf.edu/Mammography/Database.html.
} 
(a)

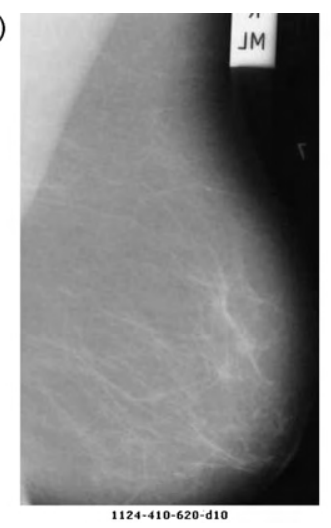

(b)

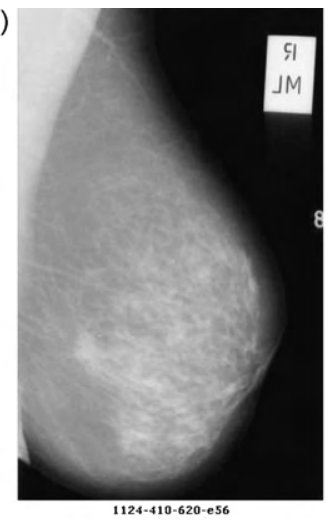

(c)

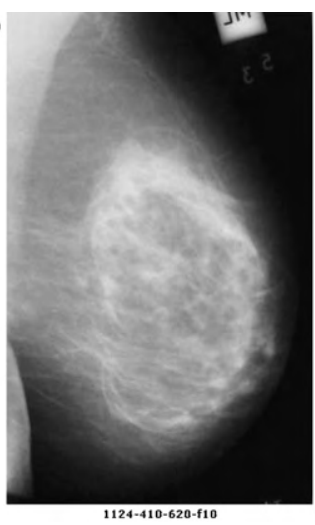

(d)

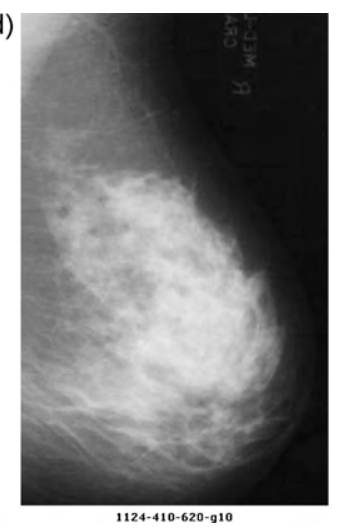

Fig. 1 - Mammographies of different breast tissues: (a) almost entirely fatty, (b) scattered fibroglandular fatty , (c) heterogeneously dense, and (d) extremely dense.

and Linear Discriminant Analysis (LDA). According to BIRADS scale, three radiologists defined the ground truth for all images. The method resulted on $60 \%, 75 \%$, and $83 \%$ of correct classification, respectively for the Naïve Bayes, Bayes, and LDA classifiers. When the number of classes was reduced to fatty and dense densities only, the results were $86 \%, 88 \%$, and $90 \%$ of correct classification respectively for the Naïve Bayes, Bayes, and LDA classifiers, indicating that coarser grouping of breast density could yield more precise results.

Histograms were used for the characterization of breast density in a set of 195 mammographies at the Medical Center of Pittsburgh by Wang et al. [13], in order to automatically evaluate breast density according to BI-RADS categories. A 71\% of correct classification was obtained with the use of a neural network classifier.

All the reported works agree with the importance of the proper characterization of breast density, as this is critical to the retrieval process. The most effective features used for characterization were extracted from the gray level histogram and texture patterns $[8,13]$. In CBIR systems, images are described as feature vectors and similarity is determined using measures of distance or as the output of classifiers that may indicate the relevance of the retrieved images to a given query. The choice of a set of features that are able to capture pictorial content in a way closer to human perception is still a challenge.

In the following section, we introduce the 2DPCA method for concise texture representation of breast densities. The method enables a CBIR system to help radiologists, by retrieving similar images for which there is a registered clinical history.

\section{Breast Density Characterization}

In CBIR systems, the access to information is performed based on the visual attributes extracted from the images. The definition of a set of features, capable to effectively describe each region of the image, is one of the most complex tasks in the process. In addition, the process of characterization affects all the subsequent steps of a CBIR system [14].
Images can be numerically represented by a feature vector, preferentially at a low-dimensional space in which the most relevant visual aspects are emphasized [15,16]. Visually, breasts of fatty and dense densities differ by gray level intensities in mammographies, as can be seen in Fig. 1. In order to describe the different patterns of parenchyma tissue within one category, the texture attribute can be used, since texture contains information about the spatial distribution of gray levels and variations in brightness, turning the representation of breast density appropriate [17]. However, the high dimensionality of a feature vector that represents texture attributes limits its computational efficiency, so it is desirable to choose a technique that combines the representation of the texture with the reduction of dimensionality, in a way to turn the retrieval algorithm more effective and computationally treatable. The two-dimensional principal component analysis (2DPCA) technique is able to satisfy these requirements.

PCA is a classic feature extraction and data representation technique that aims at finding a less redundant and more compact representation of data in which a reduced number of components can be independently responsible for data variation [16]. This method transforms a $p$-dimensional vector in another q-dimensional vector, by projecting the original vector into a lower-dimensional basis composed of $q$ orthogonal axes of maximum variance, the principal components.

Differently, 2DPCA technique [4] is based on 2D matrices rather than $1 \mathrm{D}$ vectors, as image covariance matrices can be constructed directly using the original image matrices. The idea of 2DPCA technique is to project image A, a matrix of size $m \times n$ pixels, onto $\mathbf{X}$ by the linear transformation:

$$
\mathrm{Y}=\mathrm{AX}
$$

A projected $m$-dimensional vector $\mathrm{Y}$ is obtained and defined as the projected feature vector of image $\mathbf{A}$.

In a way to get a good projection vector $\mathbf{X}$, the trace of the covariance matrix of the projected feature vectors is obtained through the adoption of the following criterion:

$J(\mathbf{X})=\operatorname{tr}\left(\mathbf{S}_{\mathrm{x}}\right)$ 
where $\mathbf{S}_{\mathrm{x}}$ denotes the covariance matrix of the projected feature vectors of the training examples and $\operatorname{tr}\left(\mathbf{S}_{x}\right)$ denotes the trace of $\mathbf{S}_{\mathbf{x}}$ :

$\operatorname{tr}\left(\mathbf{S}_{\mathbf{X}}\right)=\mathbf{X}^{T}\left[E(\mathbf{A}-E \mathbf{A})^{T}(\mathbf{A}-E \mathbf{A})\right] \mathbf{X}$

The image covariance matrix $\mathrm{G}$ of an image $\mathrm{A}$ can be defined as:

$G=E\left[(A-E A)^{T}(A-E A)\right]$

Then, the criterion expressed in (2) can be expressed by:

$J(X)=X^{T} G X$

where $\mathbf{X}$ is a unitary column vector. The optimal projection axis $\mathbf{X}_{\text {opt }}$ is the unitary vector that maximizes $J(X)$, i.e. the eigenvector of $\mathrm{G}$ corresponding to the largest eigenvalue. These optimal projections vectors of 2DPCA, $\mathbf{x}_{1}, \ldots, \mathbf{x}_{d}$ are used for feature extraction, where $d$ corresponds to the number of selected eigenvalues.

For a given image $\mathbf{A}$, let:

$\mathrm{Y}_{k}=\mathrm{AX}_{k}, \quad k=1,2, \ldots, d$

A family of projected features $\mathrm{Y}_{1}, \mathrm{Y}_{2}, \ldots, \mathrm{Y}_{k}$ is obtained, which is called principal components (vectors) of the image A. Unlike PCA, where the principal component is a scalar, with 2DPCA each principal component is a vector. The principal component vectors obtained are used to form an $m \times d$ matrix $\mathrm{L}=$ $\left[\mathrm{Y}_{1}^{\mathrm{T}}, \mathrm{Y}_{2}^{\mathrm{T}}, \ldots, \mathrm{Y}_{k}^{\mathrm{T}}\right]$, which is called the feature matrix or feature image of the image A.

Some works employed 2DPCA technique for face and palmprint representation. For instance, Zuo et al. [18] proposed an assembled matrix distance metric (AMD) to measure the distance between two feature matrices obtained through 2DPCA technique. Firstly, they used the ORL face database (1992) to evaluate the proposed technique in 400 images of size $112 \times 92$ pixels. Only the first four largest eigenvalues of the projected matrix of 2DPCA were chosen, which was the best result obtained among tests using from one to eight eigenvalues. Comparing to other image recognition methods like Eigenfaces, Fisherfaces, and D-LDA, the recognition rate using 2DPCA and AMD achieved 96.30\%, the highest one. Secondly, using the PolyU palmprint database (2004), they used 600 subimages of size $128 \times 128$ pixels to test the efficiency of the proposed method. The authors, in this case, have chosen to keep the first 18 eigenvalues after testing eigenvalues ranging from 1 to 25 . The comparison of the method was done with the three recognition methods already cited, and again 2DPCA and AMD achieved the highest recognition rate of $97.67 \%$.

Also with the aim of image recognition, Zhao et al. [19] introduced 2DPCA technique into the extraction of palmprint features, removing the illumination information using the w/o3 technique [20]. In this technique, in order to get rid of disturbance from different lightness conditions for palmprint collections and to lead to better recognition results, the first three largest eigenvalues were removed, as they were found to represent information related to illumination. From the PolyU palmprint database, the authors used 600 images, and from each one of them they extracted the central part of the palmprint. 2DPCA technique was applied, the first three principal components were discarded and PCA was used after 2DPCA to reduce dimensionality, in a process called by the authors 2DPCA(w/o3)PCA. Its performance was compared to other feature extraction techniques such as 2DGabor filter, PCA, PCA(w/o3), and LDA. 2DPCA(w/o3)PCA consumed less time for the extraction of the features and also obtained the highest accuracy rate - 99.27\% - using a classifier proposed by the authors, a modified modular neural network (MNN) classifier.

\section{Support Vector Machine for Content-Based Image Retrieval}

In this section, the support vector machine (SVM) classifier will be presented for the task of retrieval.

Image retrieval aims at retrieving, from a database, images that are relevant to a given query. The query image goes through the process of feature extraction in order to be compared to the feature vectors of all images stored in the database. The most similar images with respect to the query are retrieved and presented to the radiologist.

The support vector machine (SVM) method was developed to solve classification problems [21], but its use was extended to CBIR systems [22]. Specifically for CBIR systems of mammographies, SVM was used, for instance, by Yang et al. [23]. The SVM method is a technique that guides the construction of classifiers with good degree of generalization [5], i.e., with the ability of correctly predicting the class of a sample that was not used in the learning process. In the case of CBIR systems, SVM measures the relevance of an image to a particular query [24].

Machine learning techniques may employ an inference principle called induction, whose general conclusions are obtained from a particular set of examples. This inductive learning can be divided into two main types: unsupervised and supervised learning, which applies to SVM [16].

In unsupervised learning, there are no labeled examples. The algorithm learns how to cluster the entries according to a quality measure. In supervised learning, an external agent is used to indicate the desired answers to the entry patterns. The classifier is trained with a broad set of labeled data. In this case, given a set of labeled examples as $\left(x_{i}, y_{i}\right)$, where $x_{i}$ represents an example and $y_{i}$ denotes its label, one should be able to produce a classifier that can precisely predict the labels of the new data. This induction process of a classifier from a sample of data is called training. The obtained classifier may also be seen as a function $f$ that receives a dataset $x$ and associated labels $y$. The labels or classes represent the phenomenon of interest on which one wants to make predictions. The labels can assume discrete values $1, \ldots, p$. A classification problem in which $p=2$ is called binary.

SVM can be described for a binary classification as follows: given two classes and a set of points that belong to these classes, the SVM classifier determines the hyperplane in the feature space that separates the points in order to place the highest number of points of the same class on the same side, while maximizing the distance of each class to that hyper- 
plane. The hyperplane generated is determined by a subset of items from the two classes, called support vectors. When the set of data is linearly separable by a hyperplane, it is called a linear case of separation. The equation of an hyperplane is presented in Eq. (7), where $\mathbf{w} \cdot \mathbf{x}$ is the inner product between vectors $\mathrm{w}$ and $\mathrm{x}, \mathrm{w} \in \mathrm{P}$ is the normal vector to the hyperplane and $|b| /\|\mathbf{w}\|$ is the perpendicular distance of the hyperplane to the origin, with the bias $b \in \mathbb{R}$

$f(x)=w \cdot x+b=0$

Eq. (7) divides the space data $X$ into two regions: $\mathbf{w} \cdot \mathbf{x}+b>$ 0 and $\mathbf{w} \cdot \mathbf{x}+b<0$. A signal function $g(x)=\operatorname{sgn}(f(x))$ can be employed to obtain the classification:

$g(\mathbf{x})=\operatorname{sgn}(f(\mathbf{x}))=$

$\begin{cases}+1, & \text { if } \mathbf{w} \cdot \mathbf{x}+b>0 \\ -1, & \text { if } \mathbf{w} \cdot \mathbf{x}+b<0\end{cases}$

In most cases, however, the data set cannot be precisely separated by a hyperplane, so a function called kernel is used instead. It receives two points $x_{i}$ and $x_{j}$ from the input space, according to Eq. (9), and computes the product between these data in the feature space:

$Q\left(x_{i}, x_{j}\right)=\Phi\left(x_{i}\right) \cdot \Phi\left(x_{j}\right)$

The most commonly used kernels are the polynomial:

$\mathrm{Q}_{p}\left(x_{i}, x_{j}\right)=\left(\delta\left(x_{i} \cdot x_{j}\right)+\kappa\right)^{d}$

and the Gaussian:

$\mathrm{Q}_{g}\left(x_{i}, x_{j}\right)=\exp \left(-\sigma\left\|x_{i}-x_{j}\right\|\right)^{2}$,

where the parameters $\delta, \kappa, d$ in Eq. (10) and $\sigma$ in Eq. (11) must be preset.

For more than two classes, this problem turns into a multiclass problem $[25,26]$, which is the case of the MammoSys CBIR system. There are two basic approaches for a multi-class application. The first approach reduces the problem of multiple classes to a set of binary problems, using methods of decomposition one by class (one against all) and the separation of classes two by two (one against one).

In the one-against-all method, a SVM is built for each class through the discrimination of this class against the remaining classes. The number of SVMs used in this method is M. Test data $x$ are classified using a decision strategy, i.e., the class with the maximum value for the discriminant function $f(x)$ is assigned to the data. All the $n$ training examples are used to construct the SVM for one class. The SVM for one class $p$ is built using the set of training data $(x)$ and the desired outputs (y).

In the one-against-one method, a SVM is built for a pair of classes through training, for the discrimination of two classes. In this way, the number of SVMs used in the method is $M(M-$ 1)/2. One SVM for a pair of classes $(p, m)$ is built using training examples belonging only to these classes.
The second approach is a generalization of the binary classification to more than two classes and one of the methods that uses this approach is the method of Crammer and Singer [25]. In this method all the training examples are used at the same time.

MammoSys CBIR system is a multi-class problem that uses the one-against-one method, separating the four breast density categories two by two for classification and retrieval.

In the following section, the experiments for the development of the proposed system are presented.

\section{Experiments}

The MammoSys system was implemented using MatLab (Matrix Laboratory) through the image processing and symbolic math toolboxes, and the LIBSVM library [27]. Feature extraction was performed on an Intel Core2Quad 2.66 GHz processor with $8 \mathrm{~GB}$ of RAM under Microsoft Windows operating system and image retrieval was executed on an Intel Core2Duo $2 \mathrm{GHz}$ processor with $3 \mathrm{~GB}$ of RAM, also under Microsoft Windows operating system.

The mammographies used in this work are taken from the database of radiological images of the IRMA project that were generated using several film digitizers (the reader is referred to [7] for acquisition details). In the IRMA project, all images are coded according to a mono-hierarchical, multi-axial coding scheme [28], and this codification provides the ground truth of all mammographies, as all the images have been previously verified by an experienced radiologist. The size of the images varies from $1024 \times 300$ pixels to $1024 \times 800$ pixels. For the application of the 2DPCA technique, it is necessary that all the images have the same size. Therefore, from each image, a region of interest (ROI) of size $300 \times 300$ pixels was extracted through an automatic process, from both cranio-caudal (CC) and medio-lateral (MLO) views. This allows for the selection of regions containing only breast tissue, excluding artifacts such as annotation and exam labels from mammographies.

Although the IRMA database offers more than 10,000 images, they are not equally distributed in all categories. For instance, there are 1396 images in BI-RADS I category and 2023 images in BI-RADS IV category. So, in a way to perform the experiments more accurately without favoring a specific category, we selected a total of 5024 images. Additionally, the selection was performed so as to consider the direction (right and left) for breast imaging and the anatomy (CC and MLO) of the breast. Experiments were performed using the following number of images, for each BI-RADS breast density category and in a way to consider the direction (right and left) for breast imaging and anatomy (CC and MLO) of the breast: 314 images from CC and right breast; 314 images from CC and left breast; 314 images from MLO and right breast; and 314 images from MLO and left breast, in a total of 1256 images for each one of the four BI-RADS categories.

The methodology applied to the experiments is depicted in Fig. 2, and the steps followed for the development of the CBIR system were:

Step $1 \rightarrow$ 2DPCA feature extraction: 2DPCA technique was performed in each of the 5024 ROIs. The following principal components related to the first $d$ largest eigenvalues of the 


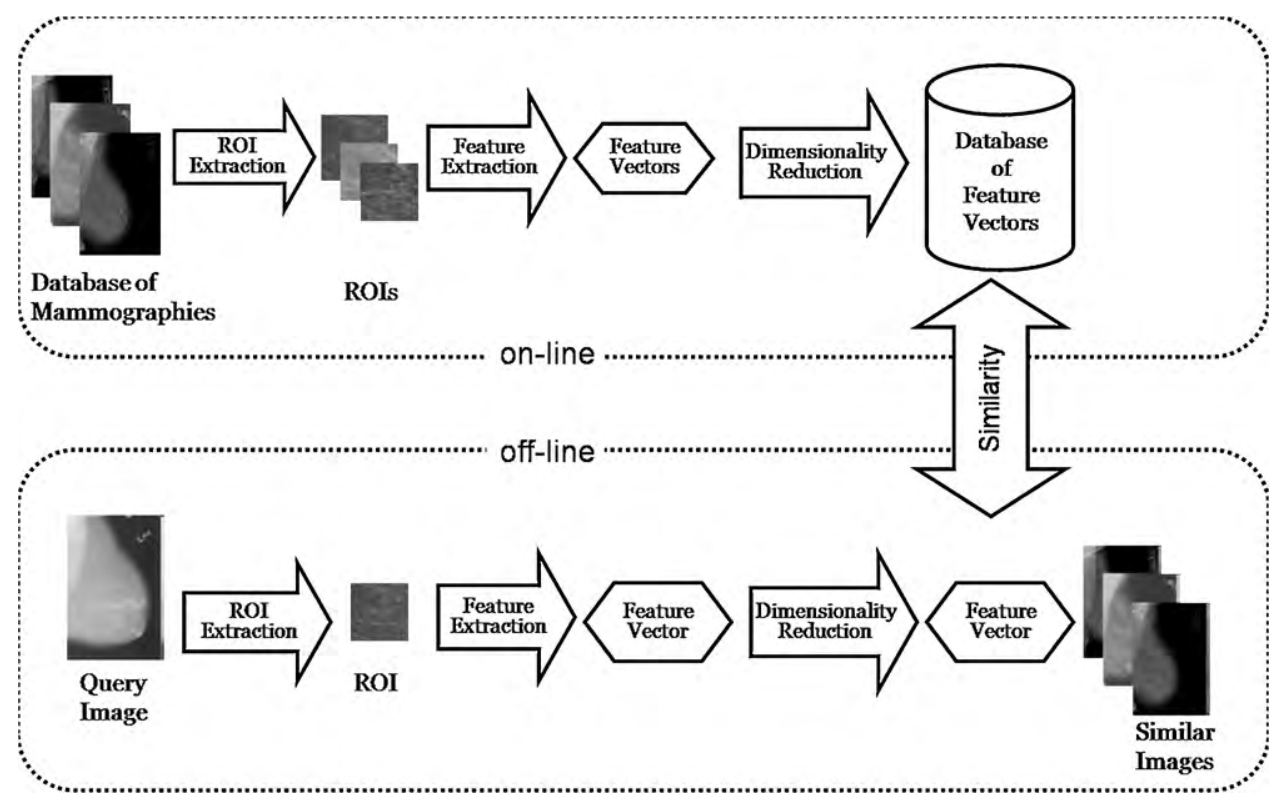

Fig. 2 - MammoSys CBIR system scheme. In the off-line stage of the system, the images of the database are processed and characterized as vectors in a lower-dimensional feature space. The on-line stage comprehends the processing of the query and the search for similar images in the database.

covariance matrix were used in the experiments: 1 to 10,15 , and 20 . These values were chosen in order for the results to be compared to previous works [18].

Step $2 \rightarrow$ Measurement of similarity between images: SVM was used to indicate the relevance of the images to a certain query. Using the LIBSVM library, that deals with unbalanced data, a 10-fold cross validation was performed and the tests were done using the polynomial kernel.

Step $3 \rightarrow$ Evaluation of the CBIR system: Measures of precision and recall were obtained and the average precision for $10 \%$ of recall was chosen, since radiologists pay more attention to the top returned images.

The performance of 2DPCA technique was compared to the ones using principal component analysis (PCA) and singular value decomposition (SVD) for breast density characterization, as these two techniques are also able to represent texture and reduce the dimensionality of the feature vector. SVM was evaluated for the task of image retrieval.

\section{Results and Discussion}

Table 1 lists the execution time of the MammoSys system, comparing the breast tissue characterization using 2DPCA, PCA, and SVD for different values of $d$. 2DPCA took more time to execute the retrieval, as expected, since each principal component is a vector, while for PCA and SVD each principal component is a scalar. Also, with $d=1, \ldots, 4,2$ DPCA was slower than for others values. Machine learning algorithms like SVM are influenced by data, i.e., the number of features may degrade computational performance. If the number of features is too small or not significant, the support vectors may not be able to correctly separate the data and indicate the relevance of the images, therefore taking more time in this task. Also, there was no significant difference between the values of the second, third, and fourth principal components of 2DPCA, and this may have delayed convergence in the computation of the hyperplane that separates data.

The average precision, for all $d$ first values, comparing the breast tissue characterization using 2DPCA, PCA, and SVD, and with SVM for the retrieval task, is listed in Table 3.

It can be observed from Table 3 that 2DPCA overcame PCA and SVD for all the values of parameter $d$. The highest value was $97.83 \%$ of average precision for the first five principal components of 2DCPA. That also, according to Table 1, was the fastest on the retrieval task. This suggests that the choice of few principal components allies images characterization with dimensionality reduction of the feature vector.

For 2DPCA technique, the average precision was not constant for all $d$ values. The insertion of more principal components could be considered as confounders, since the diagonal matrix that contains these principal components

Table 2 - Execution time, in seconds, for the CBIR

system, using 2DPCA, PCA, and SVD for breast density characterization.

\begin{tabular}{rlll}
\hline$d$ & 2DPCA & PCA & SVD \\
\hline 1 & 809.4 & 10.1 & 11.2 \\
2 & 720.8 & 10.6 & 11.4 \\
3 & 705.9 & 11 & 12.1 \\
4 & 448.2 & 11.3 & 12.8 \\
5 & 154 & 11.5 & 12.8 \\
6 & 156.1 & 12.2 & 13.9 \\
7 & 157.3 & 12.5 & 14.7 \\
8 & 167.1 & 13.1 & 14.8 \\
9 & 215.4 & 13.4 & 14.9 \\
10 & 221 & 16.2 & 18.1 \\
15 & 274.5 & 16.2 & 19.3 \\
20 & 317.9 & 18 & 21.1 \\
\hline
\end{tabular}




\begin{tabular}{|c|c|c|c|}
\hline$d$ & 2DPCA & SVD & PCA \\
\hline 1 & $83.86 \%$ & $65.93 \%$ & $67.86 \%$ \\
\hline 2 & $86.03 \%$ & $74.23 \%$ & $68.30 \%$ \\
\hline 3 & $87.96 \%$ & $77.5 \%$ & $69.05 \%$ \\
\hline 4 & $90.87 \%$ & $78.49 \%$ & $69.56 \%$ \\
\hline 5 & $97.83 \%$ & $78.27 \%$ & $69.98 \%$ \\
\hline 6 & $97.67 \%$ & $78.23 \%$ & $69.8 \%$ \\
\hline 7 & $97.09 \%$ & $78.36 \%$ & $70.39 \%$ \\
\hline 8 & $97.00 \%$ & $78.39 \%$ & $70.27 \%$ \\
\hline 9 & $96.46 \%$ & $78.63 \%$ & $70.24 \%$ \\
\hline 10 & $96.22 \%$ & $79.01 \%$ & $70.45 \%$ \\
\hline 15 & $95.50 \%$ & $80.31 \%$ & $70.44 \%$ \\
\hline 20 & $93.85 \%$ & $80.83 \%$ & $70.86 \%$ \\
\hline
\end{tabular}

has $d$ values that are significantly higher than others, and they are ordered from the highest to the lowest values. The lowest values are close to zero and can be considered insufficient to properly characterize the images, explaining the fact that average precisions decrease as the number of principal components increases. Also, the retention of few principal components may be not enough for a good breast tissue characterization. For the SVD and PCA techniques, for which the principal components are scalars and not vectors as in 2DPCA, the use of few principal components is not enough for breast tissue characterization. In this case, greater numbers of principal components will provide better average precision.

Fig. 3 shows the average precision and recall curve using the first five principal components for 2DPCA, PCA, and SVD for breast density characterization and SVM for image retrieval. The texture of breast tissue was better represented by the features extracted using 2DPCA, which was able to capture

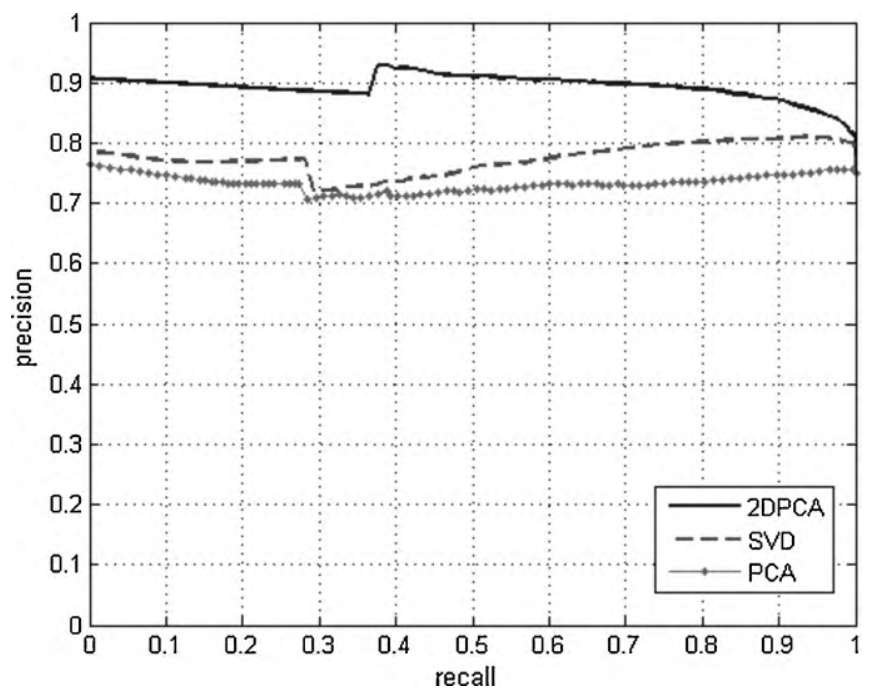

Fig. 3 - Precision $\times$ recall curve for the average precision using the first five principal components for breast density characterization, 2DPCA, SVD, and PCA for breast density characterization and SVM for image retrieval.

the difference between the gray level intensities of the breast densities. Concerning 2DPCA, for $10 \%$ of recall, a precision of $90 \%$ means that from 502 mammographies returned by the MammoSys CBIR system, 452 were relevant for the user.

Fig. 4 shows an example of a query image from breast density category BI-RADS I and retrieved images based on the first five values for the 2DPCA-SVM design. All the retrieved images are not only from the same BI-RADS category of the query image but they share the same view (CC) and direction

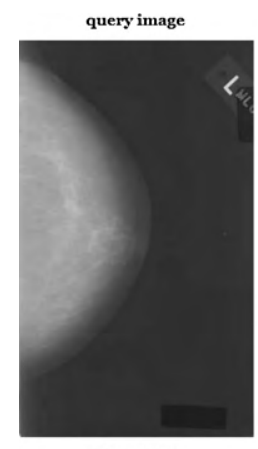

BI-RADS I retrieved 5

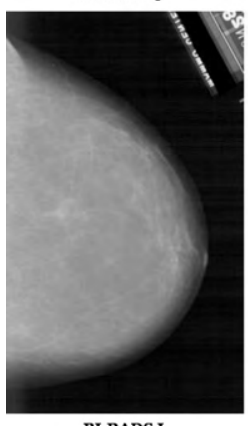

BI-RADS I

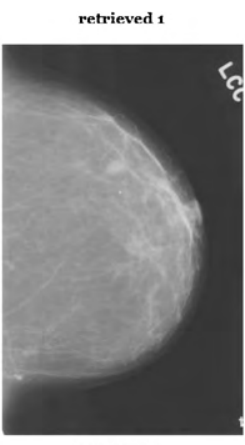

BI-RADS I

retrieved 6

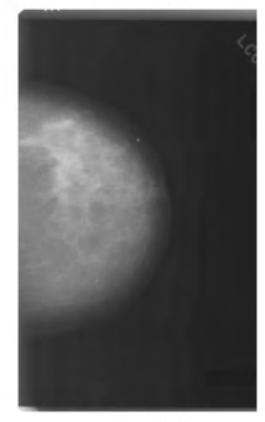

BI-RADS I

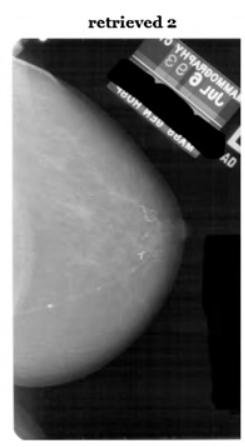

BI-RADS I retrieved 7

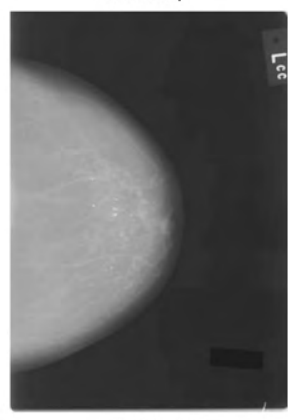

BI-RADS I

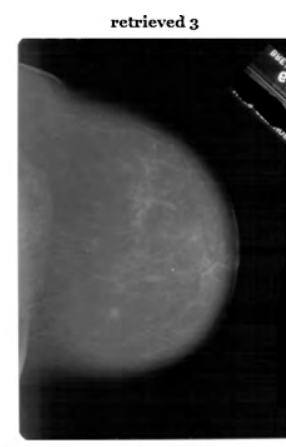

BI-RADS I

retrieved 8

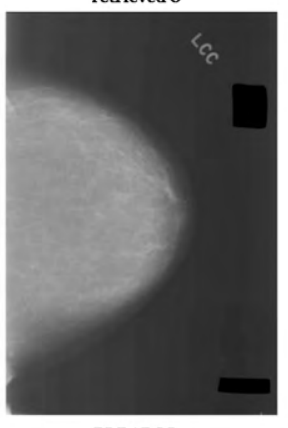

BI-RADS I

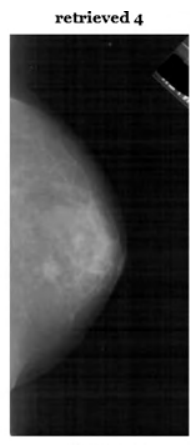

BI-RADS I

Fig. 4 - Retrieval example of one query of the MammoSys system using the first five singular values for 2DPCA and SVM with polynomial kernel. 


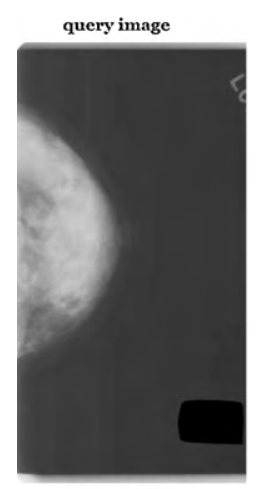

BI-RADS IV

retrieved 5

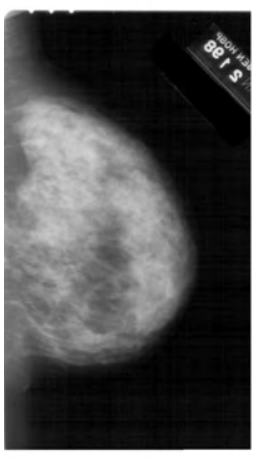

BI-RADS IV

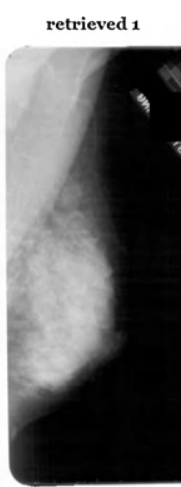

BI-RADS IV

retrieved 6

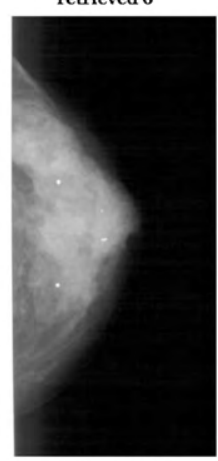

BI-RADS IV

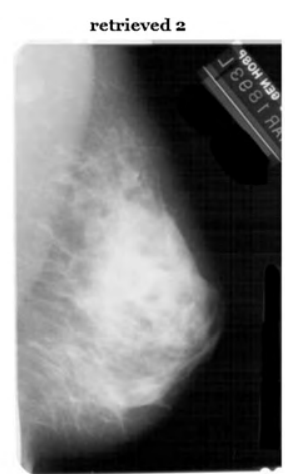

BI-RADS IV

retrieved 7

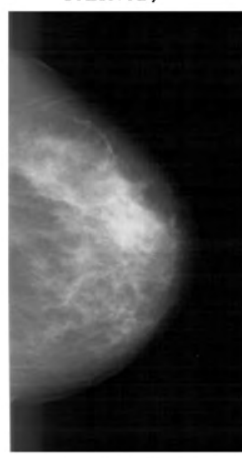

BI-RADS IV

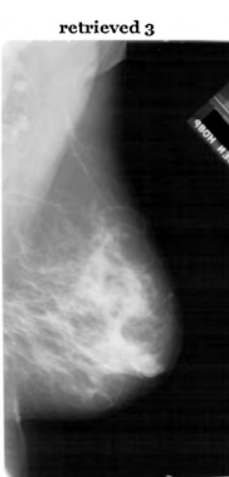

BI-RADS IV

retrieved 8

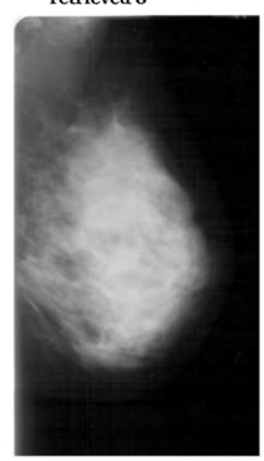

BI-RADS IV

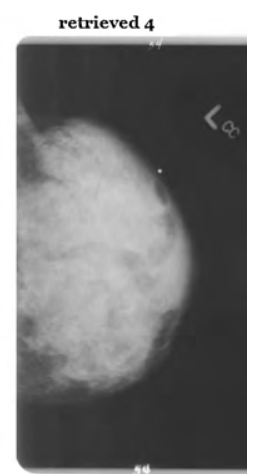

BI-RADS IV

Fig. 5 - Second retrieval example of one query of the MammoSys system using the first five eigenvalues for 2DPCA and SVM with polynomial kernel.

(right breast). Another example of retrieval is depicted in Fig. 5, using a BI-RADS IV image. Even though all the retrieved images are from the same category of the query image and from the same direction (right), they are from different views: Retrieved images 1, 2, 3, and 8 are from MLO view while the others are from CC view. This may occur due to the fact that the ROI selected from these MLO images contains a good portion of pectoral muscle that was confused with the white part of the breast density. The texture attribute was not able to differentiate these gray level intensities, for this particular BI-RADS category.

\section{Conclusion}

In this paper we presented a CBIR system that uses breast density as a pattern for image retrieval and is able to aid radiologists in their diagnosis. MammoSys can also be seen as a pre-processing stage of a CAD system for breast lesions detection. In the proposed system, ROIs containing only breast density were characterized using 2DPCA, a novel and promising method for the characterization texture in lowdimensional feature spaces. Experiments were designed to choose the best number of principal components that would be able to effectively represent texture. Furthermore, the retrieval of the mammographies was performed by SVM, enabling the development of a system that can really aid radiologists in their diagnosis. Another important characteristic of the MammoSys CBIR system is the availability of prior breast density classification, as all the images contained in the IRMA database have their ground truth already set by an experienced radiologist.

Although the results of the proposed method are superior when compared to the average precision of related works, they could be improved if texture attributes were used together with the gray level histogram, which is also able to capture differences between breast tissues. This could be done by concatenating these attributes - histogram and texture, at the feature vector, with proper weighting of their importance to breast tissue representation. Also, other similarity measures may be investigated, in a way to define the most appropriated one to this specific problem and to the feature vectors determined by the proposed method.

Future works may additionally consider other patterns for retrieval, such as breast lesions, masses and calcifications, characterized by size and shape. Breast lesions may be used together with breast density, providing a more instructive CBIR system for radiologists, as more information may become available to support diagnosis.

\section{Acknowledgements}

This work was partially supported by CNPq-Brazil, grants 306193/2007-8, 471518/2007-7, 307373/2006-1 and 484893/2007-6, by FAPEMIG, grant PPM 347/08, and by CAPES. The IRMA project is funded by the German Research Foundation (DFG), Le 1108/4 and Le 1108/9. 


\section{REFERE N C E S}

[1] J.N. Wolfe, Breast patterns as an index of risk for developing breast cancer, American Journal of Roentgenology (1976) 1130-1139.

[2] A. del Bimbo, Visual Information Retrieval, Morgan Kaufmann Publishers Inc., 1999.

[3] H. Müller, N. Michoux, D. Bandon, A. Geissbuhler, A review of content-based image retrieval systems in medical applications - clinical benefits and future directions, International Journal of Medical Informatics 73 (2004) 1-23.

[4] J. Yang, D. Zhang, A.F. Frangi, J. Yang, Two-dimensional PCA: a new approach to appearance-based face representation and recognition, IEEE Transactions on Pattern Analysis and Machine Intelligence 26 (1) (2004) 131-137.

[5] V.N. Vapnik, The Nature of Statistical Learning Theory, Springer-Verlag, New York, 1995.

[6] T.M. Lehmann, M.O. Güld, C. Thies, B. Fischer, K. Spitzer, D. Keysers, H. Ney, M. Kohnen, H. Schubert, B. Wein, Content-based image retrieval in medical applications, Methods of Information in Medicine 43 (4) (2004) 354-361.

[7] J.E.E. de Oliveira, M. Güld, A.A. Araújo, B. Ott, T.M. Deserno, Towards a standard reference database for computer-aided mammography, Proceedings of SPIE Medical Imaging 6915 (69151Y) (2008).

[8] S.K. Kinoshita, P.M.A. Marques, R.R. Pereira, J.A.H. Rodrigues, R.M. Rangayyan Jr., Content-based retrieval of mammograms using visual features related to breast density patterns, Journal of Digital Imaging 20 (2) (2007) 172-190.

[9] A. Oliver, J. Freixenet, R. Martí, J. Pont, E. Pérez, E.R.E. Denton, R. Zwiggelaar, A novel breast tissue density classification methodology, IEEE Transactions on Information Technology in Biomedicine 12 (1) (2008) 55-65.

[10] J. Suckling, The Mammographic Image Analysis Society digital datagram database, Exerpta Medica International Congress Series 1069 (1994) 375-378.

[11] M. Heath, K. Bowyer, D. Kopans, Current status of the digital database for screening mammography, in: Digital Mammography, Kluwer Academic Publishers, 1998, pp. 457-460.

[12] C. Castella, K. Kinkel, M.P. Eckenstein, P.E. Sottas, F.R. Verdun, F.O. Bochud, Semiautomatic mammographic parenchymal patterns classification using multiple statistical features, Academic Radiology 14 (2007) 1486-1499.
[13] X.H. Wang, W.F. Good, B.E. Chapman, Y.H. Chang, W.R. Poller, T.S. Chang, L.A. Hardesty, Automated assessment of the composition of breast tissue revealed on tissue-thickness-corrected mammography, American Journal of Roentgenology 180 (2003) 257-262.

[14] R. Baeza-Yates, B.R. Neto, Modern Information Retrieval, Addison-Wesley Professional, 1999.

[15] V. Castelli, L.D. Bergman, Image Databases - Search and Retrieval of Digital Imagery, Wiley-Interscience, 2001.

[16] R.O. Dudda, P.E. Hart, D.G. Stork, Pattern Classification, John Wiley Sons, 2001.

[17] R.C. Gonzalez, R.E. Woods, S.L. Eddins, Digital Image Processing using Matlab, Prentice-Hall, 2003.

[18] W. Zuo, D. Zhang, K. Wang, An assembled matrix distance metric for 2DPCA-based image recognition, Pattern Recognition Letters 27 (2006) 210-216.

[19] Z.Q. Zhao, D.S. Huang, W. Jia, Palmprint recognition with 2DPCA + PCA based on modular neural networks, Neurocomputing 71 (2007) 448-454.

[20] P.N. Belhumeur, J.P. Hespanha, D.J. Kriengman, Eigenfaces vs. Fisherfaces: recognition using class specific linear projections, IEEE Transactions of PAMI 19 (7) (1997) 711-720.

[21] M.F. Akay, Support vector machines combined with feature selection for breast cancer diagnosis, Expert Systems with Applications 36 (2009) 3240-3247.

[22] W.T. Wong, S.H. Hsu, Application of SVM and ANN for image retrieval, European Journal of Operational Research 173 (2006) 938-950.

[23] L. Yang, R. Jin, R. Sukthankar, B. Zheng, L. Mummert, M. Satyanarayanan, M. Chen, D. Jukic, Learning distance metrics for interactive search-assisted diagnosis of mammograms, Proceedings of SPIE 6514 (65141H) (2007).

[24] C.J. van Rijsbergen, Information Retrieval, Butterworth \& Co., 1979.

[25] K. Crammer, Y. Singer, On the learnability and design of output codes for multiclass problems, Computer Learning Theory (2000) 35-46.

[26] C.W. Hsu, C.J. Lin, A comparison of methods for multiclass support vector machines, IEEE Transactions on Neural Networks 13 (2002) 415-425.

[27] C.C. Chang, C.J. Lin, LIBSVM: a library for support vector machines, 2001. Software available at http://www.csie.ntu.edu.tw/ cjlin/libsvm.

[28] T.M. Lehmann, H. Schubert, D. Keysers, M. Kohnen, B. Wein, The IRMA code for unique classification of medical images, Proceedings of SPIE 5033 (2003) 440-451. 